\title{
DETECTING TRANSFER OF TRAINING THROUGH SIMULATOR SCENARIO DESIGN: A NOVICE DRIVER TRAINING STUDY
}

\author{
Wade Allen, George Park, Scott Terrace, \& John Grant \\ Systems Technology, Inc. \\ Hawthorne, California, USA \\ Email: rwallen@systemstech.com
}

\begin{abstract}
Summary: Novice drivers in comparison to experienced drivers perform poorly due to incomplete mental models of roadway hazards. This paper describes the driving simulator scenario design methods used in a novice driver training study to detect a possible transfer of training for hazard perception. Applied in a high school driver education classroom, the data of trained versus un-trained drivers is presented for pre/post-test driving scenarios, $N=67$. Results showed that while general simulator control performance between the trained and un-trained groups was similar, the trained group performed better at hazard events and exhibited fewer speeding behaviors at the post-test. Specific hazard encounters indicated that simulator training may have had an effect on performance even when the training group was not trained on the specific situation. Arguments for training transfer in hazard perception are presented.
\end{abstract}

\section{INTRODUCTION}

Previous studies have indicated that novice drivers visually scan the roadway environment more narrowly and miss more hazardous targets in comparison to experienced drivers (Mourant \& Rockwell, 1972; Pradhan, Pollatsek, Knodler, \& Fisher, 2009; Underwood, 2007). As shown in studies that remove the driving component (Underwood, 2007), novice driver inhibitions in visual scanning are not necessarily due to high vehicle control demands, but a lack of situational awareness or an incomplete mental model of roadway dangers. Analyses of driver crash data support this notion of inattention to potentially hazardous driving situations as a major contributor to the elevated crash risk of novice drivers (McKnight \& McKnight, 2003). To address this problem, past studies have introduced novice driver training programs to improve hazard perception using carefully designed birds-eye-view images of the roadway (Pradhan, et al., 2009), real-time traffic videos (Underwood, 2007), and driving simulation scenarios requiring collision avoidance (Allen et al., 2005).

The purpose of this paper is to describe the driving simulator scenario design methods used in a novice driver training study to detect a transfer of training for hazard perception in the driving environment. Presented is the data collected during a single semester at a high school driver education classroom. Two classroom periods received simulation training (trained group) while two classrooms periods did not (control group). All groups received a pre- and post-test drive on the simulator at the beginning and end of the semester.

Transfer of training is traditionally detected using a different problem context, modality or testing platform, e.g., a driver is trained on a simulator, followed by an on-road vehicle test or monitoring of real world crash rates. However, for some studies this is not always feasible. As 
such, it was hypothesized that if pre- and post-test drive scenarios could be designed such that driving hazards in both tests are matched in spatial and temporal elements, but different in driving context using simulation elements (e.g., building models, ground textures, roadway type, background scenery, etc...), priming cues to previously exposed hazard events could be minimized and evidence for training transfer could be detected. Furthermore, additional support may be found with the inclusion of a novel collision event in the post-test.

\section{METHODS}

\section{Participants}

A total of 67 driver education students (38 female, 29 male; ages $14-17$ yrs.) with signed parental consent participated in the study for a single semester as part of general curriculum course work. All participants were from a single public high school located in Manhattan, Kansas and were instructed by the same driver education teacher.

\section{Driving Simulator}

As shown in Figure 1, a single-monitor (19"), fixed-based, desktop driving simulator was used. Driver controls were Logitech PC gaming units. The simulation laboratory consisted of five simulator stations and was located adjacent to the driver education classroom.

\section{Pre \& Post Test Scenarios}

Pre/post test drives were designed to appear as different as possible using simulation elements (e.g., far horizon, building models, and ground textures), while still retaining critical testing elements. For example, both pre/post test drives were designed with a matched and counterbalanced number of intersections (9), signal light presentations ( 2 green, 1 amber), 4-way stop signs (4), speed limit zones (20, 25, 45, and 40-70 mph), traffic environments (school, residential, urban, and rural), pedestrian collision events (5), and vehicle collision events (4). Collision events were presented with the same spatial (e.g., originating from left or right) and temporal timing (i.e., time-to-collision). Furthermore, no auditory or visual feedback (e.g., crash noises or vehicle speed/position resets) was provided to drivers for poor performance (e.g., vehicle/ped collisions, missed stop signs) during both test drives. This was intended to reduce artificial increases in driver vigilance due to crash/violation alerts and maintain the integrity of the driver's behavior (good or bad) during testing. Performance feedback in terms of a run summary with listed error counts was only provided after the test drives and during the training drives for the training group students.

Specific hazard events were also designed for the pre/post test drives to detect possible group differences and training effects. Driver training was specifically provided for certain events, while for other events no training was given.

Obscured Stop Sign Event. This event involved a 4-way intersection stop with the driver's stop sign obscured by a large vehicle. The situation awareness in this event involves learning the various details associated with a four-way stop (cross street and markings) so that even though 
the stop sign is obscured, the driver still has sufficient cues to indicate a stop is required. As shown in Figure 2, the pre-assessment drive used a bus in a low speed, residential school zone to obscure the sign whereas the post-test drive used a large construction truck in a mid speed, rural town area. While training drives provided practice with four-way stop signs, at no time were they obscured by another object. Only in the pre and post test drives did this particular event appear.

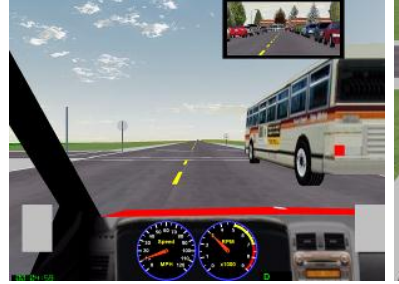

Pre-Test (school zone area)

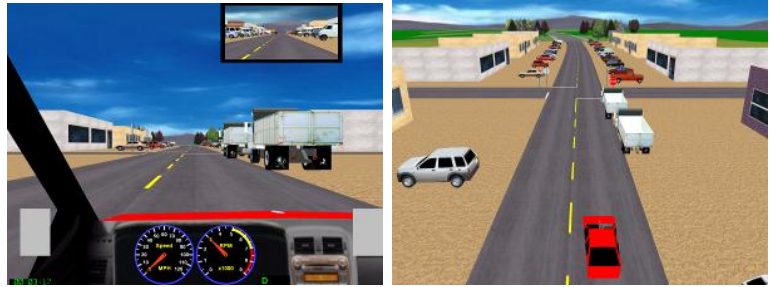

Post-Test (rural town area)

Figure 2. Obscured Stop Sign Event (driver \& aerial view) for pre- \& post-test drives

Head-On Collision Event. This event involved an oncoming vehicle passing a slower larger vehicle by entering the driver's lane. The appropriate driver reaction is to reduce speed and move to the shoulder once collision appears eminent. Specific training for this event was given in the rural training drives using the same driving contexts as in the pre-test drive. As shown in Figure 3 , the post-test drive kept a high speed rural environment but used a mountain driving scene.

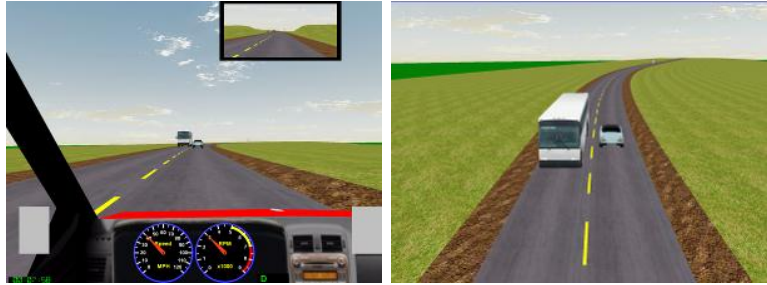

Pre-Test (prairie)

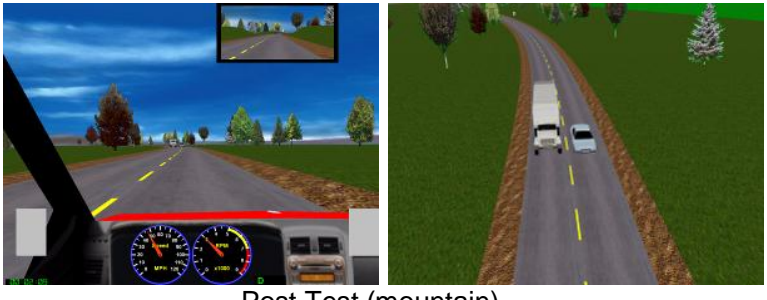

Post Test (mountain)

Figure 3. Head On Collision Event (driver \& aerial view) for pre- \& post-test drives

Vehicle Back Out Event. This event involved a perpendicularly parked vehicle backing out into the driver's path from the right, requiring a sudden stop to avoid a collision. Specific training was provided for this event in the residential training drives using the same low speed, residential environment as in the pre-test. As shown in Figure 4, the post-test drive used in contrast a midspeed, rural town environment.

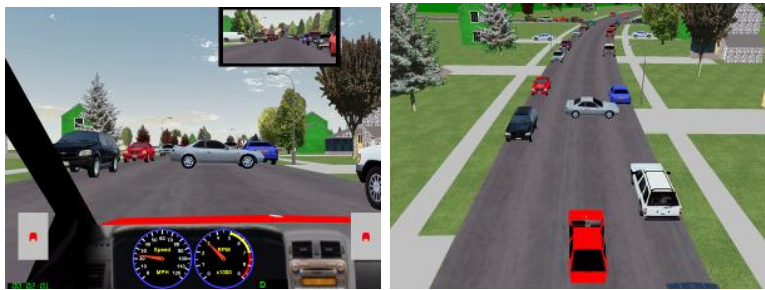

Pre-Test (residential)

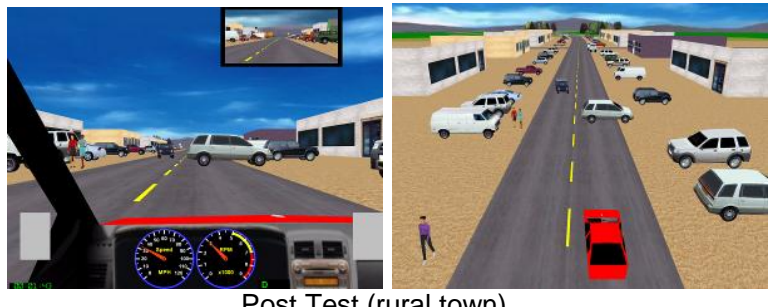

Post Test (rural town)

Figure 4. Vehicle Back Out Event (driver \& aerial view) for pre- \& post-test drives

Vehicle Backout + Head-On Event. This event was intended to provide a novel driving situation, where drivers had to put themselves into another driver's point of view. As shown in Figure 5, a white SUV on the left, backed out onto the approaching traffic lane while obscured by a larger 
yellow truck to an approaching red car. The approaching red car must then make an emergency maneuver into the driver's lane to avoid the collision. This maneuver in turn creates a head-on collision event with the driver. As the white SUV is backing out, the driver should foresee this possible outcome with the approaching red vehicle by braking early enough to allow the approaching red car's swerving behavior. While specific training was provided for vehicle backouts and head-on collisions independently, this combination event was only provided in the post-test drive and early in the scenario to reduce any event testing confounds.

\section{Testing Procedure \& Training Curriculum}

A computer-based instruction program was developed to login, track, and administer the training curriculum. As displayed in Table 1, the testing and training curriculum comprised of eight sessions (approx. 10-20 min each) with first and last sessions used for orientation and pre/post test drives. On average, students completed one session a week with groups of students entering the simulator lab during normal driver education class times.

Driving lessons were designed based on speed environments (low speed residential, mid speed urban, and high speed rural) and designed similar in appearance to the pre-test drive. Educational materials (slides and videos) of hazard situations and risky driving practices were presented at the start of each of the three lessons, followed by a short multiple choice quiz to check knowledge retention. Within each lesson, the four training drives progressed with increasing difficulty in terms of pedestrians, traffic flow, and

Table 1. Driver Training Curriculum

\begin{tabular}{clc}
\hline SESSIONS & SECTIONS & $\begin{array}{c}\text { RUNTIME } \\
(\mathrm{min})\end{array}$ \\
\hline 1 & DATS INTRODUCTION & \\
& Orientation Drive & 3 \\
& Pre-Test Drive & 6 \\
2 & LESSON 1: RESIDENTIAL & \\
& Slideshow & 11 \\
& Training Drive 1 & 6 \\
3 & Training Drive 2 & 6 \\
& Training Drive 3 & 6 \\
& Training Drive 4 & 6 \\
4 & LESSON 2: URBAN & \\
& Slideshow & 4 \\
& Training Drive 1 & 5 \\
5 & Training Drive 2 & 5 \\
& Training Drive 3 & 5 \\
& Training Drive 4 & 5 \\
6 & LESSON 3: RURAL & \\
& Slideshow & 9 \\
& Training Drive 1 & 8 \\
7 & Training Drive 2 & 8 \\
& Training Drive 3 & 8 \\
& Training Drive 4 & 8 \\
& DATS GRADUATION & \\
& Orientation Drive & 3 \\
& Post-Test Drive & 8 \\
\hline & &
\end{tabular}
hazardous events. Collision events (e.g., pedestrian crossings, vehicle pullouts) were counterbalanced to increase difficulty. Additional training scenario details have been described elsewhere (Terrace, Park \& Allen, 2010).

While training group students were given all sessions, control group students were divided into two additional groups: 1) no educational materials (sessions $1 \& 8$ only) and 2) educational materials for eight sessions. The latter group received the same slideshow materials as the simulator trained group but with additional materials in order to expose them to the simulator stations in approximately the same amount of time and frequency as the simulator trained group. Initial analyses indicated no significant differences between the two control groups and are thus treated as a single group for this paper. However, further analyses for the effect of the educational materials are warranted.

\section{RESULTS}


A multivariate analysis was conducted to assess driving performance factors by training groups (no/yes) across time (pre/post test), $N=67, \alpha=.05$. Performance factors included: collision avoidance accuracy (percentage of accidents avoided), intersection violations ( 1 amber signal and 4 stop signs), speed exceedances (posted speed limits: 20, 25, 45, and 40-70 mph), and lane keeping errors (road edge and centerline crossing counts).

An interaction effect was found for training groups and time, $F(4,62)=4.00, p=.006, \eta^{2}=.205$, suggesting that at the pre-test, no group difference was found but at the post-test, trained drivers improved more than untrained drivers. A main effect was also found for time $(p<.001)$, suggesting that both groups improved at the post-test. Follow-up univariate tests indicated interaction effects for collision avoidance $(p=.008)$ and speed exceedances $(p=.032)$. A marginal effect was found for intersection violations $(p=.070)$ and no effect for lane keeping errors $(p=.864)$. Table 2 provides the estimated marginal means (with standard deviations) for performance measures across time and training group. Significant $p$ values and effect sizes for pairwise comparisons between groups at test times are also provided.

Table 2. Driving performance measures across time and training groups

\begin{tabular}{|c|c|c|c|c|c|c|}
\hline \multirow[b]{2}{*}{$\begin{array}{c}\text { Driving Performance } \\
\text { Measures }\end{array}$} & \multicolumn{3}{|c|}{ Pre-Test } & \multicolumn{3}{|c|}{ Post-Test } \\
\hline & $\begin{array}{c}\text { No } \\
\text { Training } \\
M(S D)\end{array}$ & $\begin{array}{c}\text { Sim } \\
\text { Training } \\
M(S D)\end{array}$ & $p(\eta 2)$ & $\begin{array}{c}\text { No } \\
\text { Training } \\
M(S D)\end{array}$ & $\begin{array}{c}\text { Sim } \\
\text { Training } \\
M(S D)\end{array}$ & $p(\eta 2)$ \\
\hline Collision Avoid Accuracy & $.62(.18)$ & $.63(.18)$ & NS & $.63(.19)$ & $.80(.15)$ & $<.001(.19)$ \\
\hline Speed Exceedances & $5.8(2.9)$ & $5.9(3.3)$ & NS & $4.0(2.7)$ & $2.2(1.6)$ & $.001(.15)$ \\
\hline Intersection Violations & $1.2(1.1)$ & $1.5(1.0)$ & NS & $.91(.75)$ & $.51(.82)$ & $.060(.05)$ \\
\hline Lane Keeping Errors & $8.7(3.5)$ & $8.1(3.0)$ & NS & $7.5(2.6)$ & $6.8(2.3)$ & NS \\
\hline
\end{tabular}

NS = non significant; alpha $=.05$

While performance differences may be attributed to the trained group having more exposure to the simulator in general, the lack of difference in lane keeping errors between groups suggested that despite less exposure, untrained participants did not appear to have difficulty with vehicle controls or navigating the simulation environment. This was supported by subjective difficulty ratings ( 5 pt scale: $1=$ very easy, $2=$ =asy, $3=$ medium, $4=$ difficult, $5=$ very difficult) collected immediately after test drives. Mann-Whitney $U$ tests indicated no group differences for ratings at the pre-test $(z=-1.26, p=.21)$ and post-test $(z=-.06, p=.96)$. Wilcoxon matched-paired tests found that the trained group rated the post-test $(M=2.6, S D=.96)$ easier than the pre-test $(M=$ 2.9, $S D=.79), z=-2.13, p=.03$. Similarly, the untrained group also rated the post- test $(M=2.7$, $S D=1.13)$ easier than the pre-test $(M=3.2, S D=.81), z=-1.93, p=.05$, despite exhibiting poorer driving performance by group from the multivariate analysis. Hazard Event Analysis

A series of chi-square analyses were performed on the four hazard events (Figures 2-5) to assess whether or not group differences appeared between those students trained and untrained in the pre- and post-test drives.

Obscured Stop Sign Event. As shown in Figure 6, no disproportionate number of drivers missed or stopped at the sign between training groups at pre-test. However, group differences appeared 
for the post-test drive, with a larger percentage of drivers in the trained group (73\%) who stopped at the sign than the untrained group $(41 \%), \chi^{2}(1, N=67)=6.64, p<.05$.

Head-On Collision Event. As shown in Figure 7, the percentage of drivers who failed this event was about half for both groups in the pre-test. In the post-test; however, the trained group had no head-on collisions while over one third of the control group had head on collisions in the posttest drive, $\chi^{2}(1, N=67)=18.58, p<.001$.

Vehicle Back Out Event. No significant group differences were found for both pre/post test drives. As shown in Figure 8, almost all drivers $(95+\%)$ were able to successfully avoid this particular collision event. Set with a time to collision of 3 seconds, the designed event of a vehicle backing out appeared to be too easy for beginning drivers.

Vehicle Backout + Head-On Event. Provided only in the post-test drive, a larger proportion of drivers in the training group (67\%) were able to avoid a collision in this situation than the control group $(36 \%), \chi^{2}(1, N=67)=5.53, p<.05$. As shown in Figures $7-9$, despite high performance in the head-on collision and back-out events, $64 \%$ of untrained drivers were not able to avoid a collision when these events were combined in a novel situation.
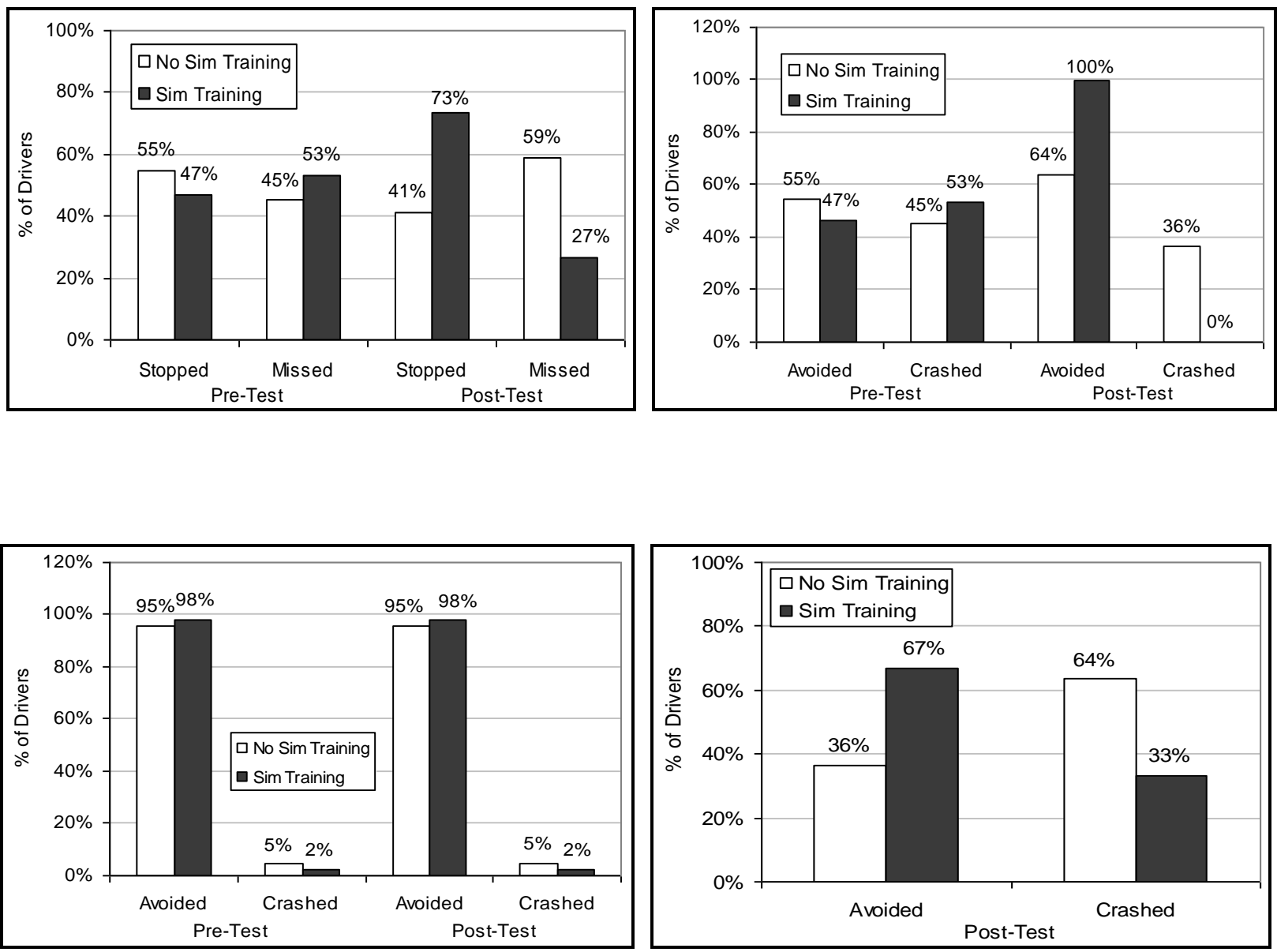


\section{DISCUSSION}

Provided in this paper was a description of the data collected and the scenario design methods used for assessing the training efficacy for a novice driver training program. Results suggested that training groups did not differ significantly in driving performance during the pre-test drive; however, group differences emerged at the post-test. Participants in the training group provided better collision avoidance accuracy and fewer speed limit violations. A marginal difference was found for intersection violations and no difference was found for lane keeping errors. Analysis of specific hazard events also confirmed no group differences at the pre-test, but apparent during the post-test with trained drivers performing better than untrained drivers in regards to detecting a hidden stop sign and avoiding a head-on collision event. No group differences were found for the vehicle backout event, but this may have been due to an inadequate time-to-collision setting or the fact that this event was largely a simple brake reaction time event. Although both groups were able to handle the vehicle backout event, when it was combined in a novel manner with a head-on collision event and the driver had to extrapolate the event towards another driver's perspective, much fewer untrained drivers appeared to be able to avoid the situation without a crash. This suggests that with repeated exposure to backouts and head-on collisions (in training sessions), trained drivers may have used or had access to a larger mental schema of the situation and that meta-training (i.e. gaining experience in hazard perception and decision making on other tasks) may have occurred.

While it can be argued that the observed performance differences were due to one group having more simulation exposure time, this paper provided evidence to the contrary. If it were the case that the untrained group merely performed worse due to lack of simulation exposure, we would expect to see differences in vehicle control demands. Instead, the data provided no differences in vehicle lane keeping, no decreases in vehicle speed to indicate higher cognitive workloads, and low subjective difficulty ratings of the scenarios. Furthermore, despite having poorer performance in the post-drive, the untrained group (similar to the trained group) rated the posttest as easier than the pre-test. Since performance feedback during the tests was removed, subjective ratings in conjunction with performance measures indicated that the untrained group exhibited a lack of hazard awareness during the test drives.

Despite suggestive findings, this study has several limitations. For actual confirmation of the program's training efficacy, future studies will require cross-platform methods for assessing hazard perception abilities (e.g., driving films, on-road testing, and longitudinal crash data). Additional analyses are also required for the effect of the educational materials presented to the students as well as any inherent differences in classroom periods. Future novice driver training studies may also do well to incorporate a more mixed methods approach by incorporating focus groups or individual interviews with students/instructors to further develop salient driving scenarios and provide a more comprehensive picture of how novice drivers perceive and react to hazardous driving situations.

\section{ACKNOWLEDGEMENTS}


Systems Technology, Inc. is a privately-owned company that manufactures and distributes the STISIM Driving Simulator used in this study. This study was funded by the Center for Disease Control and Prevention (Grant No. 1R43CE001492-01). The authors are thankful for the support of Manhattan High School, Kansas and the efforts of the driver education teacher, Brad Willie (pictured in Figure 1).

\section{REFERENCES}

Allen, R. W., Cook, M. L., Park, G., Rosenthal, T. J., Fiorentino, D. D., \& Viirre, E. (2005, January 9-13,). A PC Based Simulation System for Driver Assessment and Training. Paper presented at the TRB Annual Meeting, Washington, D.C.

McKnight, A. J., \& McKnight, A. S. (2003). Young novice drivers: careless or clueless? Accident Analysis and Prevention, 35(6), 921-925.

Mourant, R. R., \& Rockwell, T. H. (1972). Strategies of visual search by novice and experienced drivers. Human Factors, 14(4), 325-\&.

Pradhan, A. K., Pollatsek, A., Knodler, M., \& Fisher, D. L. (2009). Can younger drivers be trained to scan for information that will reduce their risk in roadway traffic scenarios that are hard to identify as hazardous? Ergonomics, 52(6), 657-673.

Terrace, S., Park, G.D. and Allen, R.W. (2010), “A Novice Driver Training System,” Presented at the IMAGE 2010 Conference, Scottsdale, Arizona.

Underwood, G. (2007). Visual attention and the transition from novice to advanced driver. Ergonomics, 50(8), 1235-1249. 